Accepted (peer-reviewed) version of article

It is authorized for self-archiving after an embargo period of 24 months.

Formatted by the author to enhance readability. 
Published in Journal of Archaeological Science 61 (2015) 287-288.

\title{
Book review: Dynamics of Learning in Neanderthals and Modern Humans. Vol. 1, Cultural Perspectives, edited by Takeru Akazawa, Yoshihiro Nishiaki, and Kenichi Aoki. Vol. 2, Cognitive and Physical Perspectives, edited by Takeru Akazawa, Naomichi Ogihara, Hiroki C. Tanabe, and Hideaki Terashima. Springer, 2014.
}

\author{
Frederick L. Coolidge ${ }^{1,2}$ and Karenleigh A. Overmann ${ }^{2,3}$
}

Dynamics of Learning in Neanderthals and Modern Humans is a two-volume proceedings of an international conference held in Tokyo in 2012 on the replacement of Neandertals by modern humans. Admirable in its multi-disciplinary scope and depth, the series represents an ambitious inquiry into the cultural, psychological, neuroscientific, and physical differences between the two species that may have contributed to the Neandertal demise. One of the key hypotheses is that differences in learning abilities might explain the replacement of Neandertals by modern humans. Thus, many of the papers in the first volume examine learning strategies and behaviors in human groups, both prehistoric and extant hunter-gatherer societies, though not within similar cognitive paradigms. Other papers review aspects of cultural evolution, including evolutionary rates of cultural change, niche construction, and innovation. The second volume continues with studies of cognition and psychology, including papers on individual, imitative, and instructive learning, problem-solving, and cognitive flexibility, as well as genetic studies, issues associated with reconstructing fossil crania, and brain morphology.

A few of the more provocative papers appear at the beginning of each volume, and they highlight an issue critical to the question of what happened to the Neandertals: Did something about Neandertal physical characteristics, brain morphology, or cognitive capacity (including cognitive flexibility) contribute to their demise? Closely related is the question of whether it was something about Homo sapiens physicality, brains, or cognition. Or, might the Neandertal extinction have been a simple twist of fate? João Zilhão (Vol. 1, Chapter 3) endorses the latter position on the highly debatable grounds that Neandertals and modern humans had identical symbolic cultures. In Zilhão's view, Neandertal- $H$. sapiens indistinguishability can only be rejected by "distortions of method and logic" and "double standards in the assessment of the evidence” (p. 52). The false dichotomy is obvious: Either Neandertals were indistinguishable from $H$. sapiens, or those identifying Neandertals as different from $H$. sapiens are bad scientists pursuing outdated and racist agendas.

Unlike humans around the globe today, who possess highly similar brains but widely divergent material cultures, Neandertals and contemporary $H$. sapiens had morphologically distinct brains and similar but not identical archaeological signatures. Sensitivities around discussions of the former (Bloch, 2012) have perhaps straightjacketed discussions of the latter. Zilhão dismisses the attempt to infer cognitive distinctions from differences in the two archaeological signatures as "a persistent, if subconscious influence in academia of Victorian-age ideas of evolution-as-progress and ancient-as-primitive” (p. 52). Notably, this contention not only minimizes asymmetries in the quantity and degree of behaviors like art, ornamentation, and ritual burials between the two species, it also ignores $H$. sapiens' plastic adaptability in

\footnotetext{
${ }^{1}$ Psychology Department, University of Colorado, Colorado Springs. Corresponding author: fcoolidg@uccs.edu.

${ }^{2}$ Center for Cognitive Archaeology, University of Colorado, Colorado Springs

${ }^{3}$ School of Archaeology, University of Oxford
} 
exploiting divergent climates and resourcing situations, perhaps the characteristic that ultimately allowed it to flourish into today's globally disbursed cultural mosaic. The forum's willingness to tackle such controversy head-on and incorporate perspectives arguing against its very enterprise shows a commendable flexibility and inclusiveness.

Another thorny issue concerns the nature of symbolism and symbolic culture. While the archaeologists and neuroscientists involved in the series were scrupulous in documenting their methodologies and statistical analyses, they unfortunately often failed to define these critical concepts in clear and unambiguous terms, or operationalize them so that they can be discerned in the archaeological record. An exception is the article by Steven Mithen (Vol. 2, Chapter 2), which addresses the use of pigment and its implication in symbolic thought (though Mithen also introduces the term " $H$. neanderthalensis symbolism”" [p. 10] without elucidating what the term might mean, its neurological underpinnings, or how it might differ from symbolism as practiced by $H$. sapiens). To Mithen's further credit, he also raises an important middle position in the false dichotomy: It is possible that Neandertals and $H$. sapiens were different from one another, without the differences necessarily implying that one species should be judged as better than the other, the other one worse.

One of the more promising studies in the series, particularly in regards to brain morphology, is that of Daisuke Kubo and colleagues (Vol. 2, Chapter 24). There is a burgeoning literature on the role of the cerebellum in higher-order cognitive functions, particularly abstraction and creativity. While the study did not include an examination of Neandertal skulls, the authors describe a new method of estimating cerebellar volume from the MRIs of 32 Japanese subjects. This study is critical because to date only Anne Weaver $(2001,2005)$ has claimed that the cerebellum-to-cortex ratio was larger in $H$. sapiens than Neandertals. Though Kubo et al. disagreed with Weaver's measurement methods, they confirmed that a depression in the posterior fossa of extant skulls was highly correlated with cerebellar volume, a finding that seems extensible in principle to fossil crania. Given the evidence of cerebellar involvement in many higher cognitive functions, the findings have significant implications for examining how brain morphology might have contributed to the evolutionary success of $H$. sapiens.

Other noteworthy contributions include the articles by Hideaki Terashima and Jun Takakura. Terashima (Vol. 1, Chapter 8) examined the evolutionary development of learning and teaching strategies of modern hunter-gatherers. Particularly insightful was his analysis of the interaction between social systems, biological and ecological systems, and cognitive systems upon learning performance. Particularly relevant to the Neandertal- $H$. sapiens debate was the emphasis on widespread social networks and how the connectivity between people in huntergatherer societies brings the opportunities for exchange of material goods and ideas. Further, he suggested that range of mobility and social networking may shed light on Neandertals' ability to innovate. Takakura (Vol. 1, Chapter 9) analyzed refitted sets of lithic artifacts from various Upper Paleolithic sites throughout Japan. His careful and extensive analyses provide welcome new insights into the pedagogical techniques of expert stoneknappers. By examining the refitted artifacts and associated debitage, Takakura was able to conclude that experts taught blade production to their novices by instruction, as well as observation and imitation. These studies, as is true of many in the series, promise novel avenues of research for understanding the different fates of the two human types. 


\section{References}

Bloch, M. (2012). Anthropology and the cognitive challenge. Cambridge: Cambridge University Press.

Weaver, A. H. (2005). Reciprocal evolution of the cerebellum and neocortex in fossil humans. Proceedings of the National Academy of Sciences, 102(10), 3576-3580.

Weaver, A. H. (2001). The cerebellum and cognitive evolution in Pliocene and Pleistocene hominids. Ph.D. dissertation, University of New Mexico. 